\title{
APRENDIZAJE ORGANIZATIVO Y GENERACION DE COMPETENCIAS
}

\section{Florentina Moreno Peláez}

Universidad de Valladolid

\section{RESUMEN}

El Aprendizaje Organizativo es algo más que la generación de competencias en los miembros que actúan en la organización. El AO se materializa, como tal, en innovaciones organizativas (métodos, tecnologías, cultura, productos). Con ello se consigue mejorar resultados y potenciar la relación dialéctica con el entorno, aumentando sus expectativas de supervivencia y desarrollo en el tiempo. Para conseguir estos incrementos se debe dar un tipo de aprendizaje que llamamos organizativo-institucional, cuyo resultado es un "espacio cooperativo" superador de las barreras organizativas y de la lógica competitiva interna que parcela saberes y los convierte en base de poderes particulares. Son precisos, por tanto, dos enfoques metodológicos para abordar el problema: el pensamiento sistémico y el dialéctico.

\section{DIMENSIONES DEL APRENDIZAJE ORGANIZATIVO}

El aprendizaje organizativo es un enfoque de gestión inexcusable en un entorno tan altamente competitivo, complejo e incierto como es el actual. Es la herramienta fundamental para conseguir ventajas competitivas, en tanto que el aprendizaje potencia la inteligencia, adaptativa y creativa, en los individuos $y$ en las organizaciones.

Entendemos por inteligencia la capacidad de un sujeto -individual o colectivo- para relacionarse con un entorno de la forma mejor posible para el

\section{Reis}


despliegue de sus facultades y la realización de sus objetivos. Es un tipo de relación dialéctica, por la cual el sujeto, afectado por una serie de condicionantes, que se le presentan como obstáculos y/o recursos, evoluciona y cambia, al tiempo que incide en los mismos condicionantes. Es, pues, un proceso abierto al tiempo y al espacio, en el cual el tiempo y el espacio son a la vez variables referenciales y resultado.

Memoria histórica de lo aprendido, perspectiva espacial o ubicación del sujeto, capacidad de aprender, es decir, de cambiar ante la retroalimentación del entorno, y visión de futuro deben ser articulados en esa capacidad autorreflexiva y operativa que llamamos sujeto o inteligencia.

Este preámbulo nos indica que entendemos el aprendizaje organizativo como algo diferente a la adquisición individual de competencias y de rutinas organizativas, aunque las incluya.

Para mejor situarnos en el tema, y teniendo en cuenta la profusión de términos con los que se definen estos procesos, vamos a revisarlos.

De la literatura organizativa anglosajona procede el término más conocido de "Organizational Learning", con el que se refieren tanto al aprendizaje individual que se deriva de la experiencia organizativa como a la repercusión que el aprendizaje tiene en los procesos organizativos, que generan cambios en la organización. No obstante, el término "The Learning Organization» hace referencia a la organización como la entidad que aprende. Es la traducción del concepto manejado en la literatura europea cuando trata de la organización que se autocualifica: "Organización cualificante» (Stahl y otros, 1993), "Organisation qualifiante» (Comisión de las CC.EE., 1993), lo cual supone a su vez una "Organisation qualifiée» (Educación permanente, 1992). Más expresamente diferencian ambos procesos como "Organisation formatrice» y "Organisation apprenante» (ADITE, 1995). También las publicaciones alemanas distinguen entre "Lernen in der organisation" y "Lernen der Organisation" o "Lernenden Organisation" (Geissler, 1991).

A la organización capaz de aprender y de autocualificarse se le denomina también "organización inteligente» (Landier, 1991). Es una organización abierta al aprendizaje y capaz de gestionar su propio cambio (Senge, 1990; Sérieyx, 1993, o Peters, 1992, por citar algunos, ya que todas las nuevas propuestas de modelos organizativos contemplan esta capacidad de adaptación al continuo cambio del contexto).

En español se han traducido los términos «Organizational Learning» y Learning Organization indistintamente por "Aprendizaje organizativo" y por "Aprendizaje organizacional", seguramente por la influencia del término "Desarrollo Organizacional», de uso generalizado, sobre todo en el contexto hispanoamericano, desde los años sesenta, si bien la utilización correcta del castellano no lo permitiría. 


\section{APRENDIZAJE ORGANIZATIVO: SU VALOR AÑADIDO}

Nosotros haremos un uso diferenciado de ambos términos. Hablaremos del aprendizaje organizativo para referirnos a los procesos de generación de nuevas competencias en los miembros que actúan en la organización, aprendiendo de la experiencia organizativa y de la actividad expresamente formadora de la organización; así como del aprendizaje, que, en la forma de nuevos saberes técnicos, nuevos productos y nueva cultura organizativa, emerge en el conjunto de la organización globalmente considerada. Son dos caras de una misma moneda, lo uno no puede darse sin lo otro, pero son diferentes.

Es un tipo de aprendizaje que se evidencia como proceso en las situaciones de cambio de algún elemento sustancial. La gestión del proceso muestra el valor añadido que implica el aprendizaje organizativo, en sus tres vertientes: el aumento de las capacidades profesionales y personales de los partícipes, el aumento de "productos» propios, como métodos nuevos de trabajo, saberes técnicos específicos, tecnologías innovadoras, etc., y el aumento de las expectativas de supervivencia y desarrollo de la organización, a través de mejores resultados, mejor posicionamiento en el tiempo y en el espacio, mayor capacidad de adaptación, etc.

Normalmente, en esos procesos se centran los estudios empíricos y las propuestas metodológicas específicamente organizativas. No obstante, la experiencia de fracasos más que de éxitos nos enseña que la integración de los dos niveles de aprendizaje - individual y global - exige un tercer nivel, que actúa de mediador entre ambos y que es de naturaleza distinta. Es un aprendizaje acerca del fenómeno organizativo, eminentemente social y sociológico, por no decir político. Es a ese proceso al que vamos a llamar aprendizaje organizacional, que, estrictamente hablando, supone aprendizaje organizativo-institucional.

\section{APRENDIZAJE ORGANIZACIONAL E INTELIGENCIA ORGANIZATIVA}

Su valor añadido será la inteligencia organizativa, que implica, en primer lugar, saber fundamentar sus necesidades de cambio en un contexto cada vez más extensao y profundamente conocido y comprendido (adecuada elaboración y difusión de estrategias).

En segundo lugar, saber cambiar su sistema integrador de competencias y recursos, a partir de la comprensión de la naturaleza de todas las variables intervinientes, en su doble cualidad de causa y efecto.

El ajuste de estas dos funciones sistémicas, adaptación e integración, es eminentemente dialéctico, arrastrando consigo esa confrontación una lógica o racionalidad que supera la propiamente sistémica. La resolución dialéctica o superadora de las contradicciones organizativas que ese contraste funcional pone en evidencia potenciará la función productiva del sistema organizativo. 
Esta inteligencia la consiguen evidentemente los sujetos, pero sujetos que están en relación y cuya relación determina la comprensión de los mismos procesos a comprender. Las organizaciones son marcos de significación no homogénea, significación que hay que interpretar y elucidar.

Y, por ultimo, el valor añadido más importante es que el aprendizaje organizativo se hace posible gracias, como hemos dicho, a la función intermediadora entre el individuo y la organización.

\section{PLANOS DE EMERGENCIA DE LA INTELIGENCIA ORGANIZATIVA}

No toda conducta de aprendizaje en la organización o de la organización es inteligente desde el punto de vista organizativo. Para que la inteligencia organizativa se dé es necesario un nivel de aprendizaje que supone perspectiva sobre los condicionantes concretos y particulares.

Hay que salirse del tiempo presente para, teniendo en cuenta el pasado, tanto de la organización particular como del fenómeno organizativo, plantearse el futuro en un contexto hipotético, pero en el que el fenómeno organizativo incide gravemente. Hay que salirse de los marcos de referencia organizativos y mentales al uso en la organización para cuestionarlos desde un plano superior, que en parte resulta del diálogo entre la diversidad particular y, en parte, de la toma de conciencia del plano de universalidad y globalidad que exige ese diálogo.

El aprendizaje organizativo propiamente dicho exige la interfuncionalidad $y$ la transferencia de aprendizajes entre diferentes departamentos, diferentes niveles jerárquicos, diferentes disciplinas o especialidades, diferentes personas... Eso exige saltar barreras organizativas, intereses particulares, valores concurrenciales, hábitos personales, modelos de autoridad, lenguajes inconvenientes, sistemas de reconocimiento tradicionales — que se han convertido en derechos-, sistemas de retribución institucionalizados y rígidos, y hasta posiblemente sistemas de contratación o de vinculación de los miembros a la organización. Sin olvidar el factor más determinante: el tipo de poder y su reparto, así como el sistema de normas, explícitas e implícitas, que lo soporta.

Sin poder es imposible promover el aprendizaje organizativo, y el poder, a su vez, puede ser - y de hecho lo es- su mayor obstáculo. Y no hablamos sólo del poder que se deriva de la propiedad del capital, sino de los poderes estructurales, bien apalancados en tareas de control, que en su día pudieron ser sistémicamente funcionales, dado el carácter del sistema productivo, pero que hoy son altamente disfuncionales, e implican que se han apropiado, de hecho, de las organizaciones. 


\section{EL OBJETO DEL APRENDIZAJE ORGANIZACIONAL: EL ORGANIZARSE}

El objeto, pues, del aprendizaje organizacional es la misma organización, su sustrato constitutivo, algo que es a la vez evidente y oculto. Toda realidad social constituida afirma un modo de ser y niega otro modo posible y alternativo. Las necesidades de cambio ponen en evidencia esa doble función de lo establecido y potencian su dialéctica. De este modo la organización se «desorganiza», permitiendo que emerja el organizarse: el aprendizaje organizativo.

El aprendizaje organizacional supone una toma de conciencia organizativa, aprendizaje teórico y político, que se hace operativo en un cambio en el poder, en las reglas, en los valores y en la finalidad de la organización, que se dimensiona.

En la práctica, a partir de las características, posicionamiento y estrategias de la organización, así como del análisis del contexto, podemos empezar por el diagnóstico de las necesidades competenciales y organizativas al nivel operativo. Del análisis comparativo entre necesidades y recursos obtendremos el camino a recorrer aprendiendo y gestionando las competencias necesarias. El camino también nos mostrará la naturaleza de los procesos y los instrumentos congruentes. La organización puede, sin muchas dificultades, gestionar su papel formativo expreso, cuando las necesidades se satisfacen con capacidades técnicas de sus miembros. Su papel formativo es más difícil cuando necesita conductas innovadoras, que dependen más de la voluntad que de la capacidad. Pero lo más problemático es que la propia organización aprenda y que aprenda a aprender. Es decir, que surja el organizarse y que se haga consciente a todos los niveles. Sólo así cada parte puede entender la necesidad del cambio, participando en su planificación y realización con «conocimiento de causa».

\section{EL ORGANIZARSE Y LA ORGANIZACION: LA VINCULACION DE LOS ACTORES}

Estamos planteando un condicionante básico y sustancial para el aprendizaje organizativo: la implicación de los sujetos, en tanto que individuos concretos y en tanto que colectivos que deben actuar como unidad funcional.

La implicación del individuo puede conseguirse, siguiendo la lógica o racionalidad instrumental operante en la organización, a través de ventajas de tipo económico, de status y personales. En ese nivel la motivación es coherente con el sistema de valores de la organización, normalmente refrendado a nivel social, dado que los resultados son percibidos, todavía, como exitosos.

El problema se complica cuando tengo que configurar espacios de implicación cooperativa, que están en contradicción con la asignación de ventajas individuales, o departamentales, si considero a la organización en su conjunto. Los 
mecanismos integradores que se derivan de la división del trabajo ya no sirven. La división del trabajo - la especialización y los diferentes niveles de responsabilidad - quizá siga siendo necesaria técnica y socialmente hablando, pero debe ser superada para que aparezca la implicación cooperativa.

Esto supone que el conjunto, el grupo, la organización, la sociedad, la humanidad... deben aparecer como la referencia. Así, pues, valores, racionalidad, finalidad institucional, poder y legitimidad y objetivos tienen que replantearse para modificar las expectativas de ventajas a conseguir con la implicación.

\section{NATURALEZA DE LA ORGANIZACION INTELIGENTE}

El problema del aprendizaje organizativo ha puesto en evidencia la verdadera naturaleza de los procesos organizativos: su hipercomplejidad, su dinámica y tensión interior, su ambigüedad e incertidumbre, su funcionalidad paradójica, sus contradicciones... y su fuerza vital y creadora. Algo que no se puede gestionar por simplificación y control. Si simplifico y controlo, gestiono otra cosa, un artefacto.

La cooperación social es, efectivamente, el único mecanismo de aprendizaje organizativo y de desarrollo de capacidades individuales y de relación social (Piaget, Fromm, Mead, Bandura, etc. ). Los espacios de cooperación social —inteligentes - son capaces de superar la enajenación del hombre, individuo y especie, respecto de su propia autogestión en el entorno - ahora ya universal- en el que se realiza.

La cooperación para conseguir un fin legítimo es una pauta de comportamiento, por otro lado, intrínsecamente necesaria y muy satisfactoria. El trabajo humano significa mucho más que lo que significan las ventajas instituidas como tales, si al trabajo le volvemos a dar su capacidad generatriz de productos a compartir (culturales, sociales, económicos), de capacidades humanas a desarrollar, y de toma de conciencia social y ética.

Conseguir esto en los contextos organizativos concretos supone un nuevo tipo de organización. Es un cambio revolucionario posible.

Podríamos prestar atención, como posible referencia, a la evolución de las «Empresas del tercer sector» (ONGs, asociaciones, voluntariado...), como empresas más capaces de conjugar la lógica de la solidaridad con la lógica instrumental, dado su origen y finalidad, así como el tipo de vinculación de sus miembros.

Por el momento, es verdad, ni las organizaciones del primer sector, cuya referencia es el mercado y cuya lógica operativa es únicamente la económicoinstrumental y concurrencial; ni las del segundo sector o públicas, orientadas a la gestión del común, pero demasiado burocratizadas; ni las del tercer sector, cuya lógica es la solidaridad, pero que no han encontrado la adecuada integra- 
ción con sistemas de gestión racionalizados, han sido hasta ahora capaces de generar aprendizajes generalizables que pudieran servir de modelo. Tenemos, pues, que hacer camino al andar, para tratar de conjugar criterios sociales y eficacia en la gestión.

\section{EL PROCESO DE CONFIGURACION DE ESPACIOS DE COOPERACION SOCIAL}

Construir ese espacio normativo capaz de determinar el comportamiento hacia la cooperación es el principal desafío. No bastan las normas legales. La percepción cambiante y diversa del bien común y de las condiciones objetivas modifica los criterios generales de legitimación, que luego se han de conjugar con los intereses particulares. De ahí que las expresiones culturales de los grupos, las diversas culturas y subculturas, cobren cada vez más importancia, así como la dimensión subjetiva de la acción social.

Intentaremos bajar al terreno empírico, aunque esto suponga un corte de estilo, que no de contenido, para poner algunos ejemplos.

\section{La transversalidad}

Estamos trabajando en una organización del primer sector - una empresa industrial — , que ha implantado el sistema de gestión y organización del trabajo de Calidad Total. Se ha desplegado todo un sistema de formación en profundidad, para dar a conocer no sólo el modelo, sino el "Proyecto Empresa», que ha sido participado a todos los niveles, desarrollando a su vez las capacidades que el nuevo modo de trabajar exige. El estilo de mando debe ser proactivo, impulsor, capaz de generar la comunicación, capaz de formar, de negociar, etc. De hecho, ha habido muchos aprendizajes en esta línea. Pero no emergen comportamientos transversales, es decir, de cooperación entre diferentes departamentos, diferentes unidades básicas, diferentes niveles jerárquicos, diferentes "posicionamientos» individuales y de grupo. Existen acciones transversales institucionalizadas y sistematizadas, pero la Calidad Total o la Mejora continua exigen la transversalidad del día a día. En el día a día se da algún comportamiento transversal que pertenece a la cultura de la organización desde siempre y que llaman «compadreo»; es, pues, una transversalidad selectiva a criterio personal, informal.

\section{Los espacios para el debate: la crítica}

Puestos a analizar el problema con un grupo de mandos intermedios voluntarios, podemos ver cómo se perciben los obstáculos. Un obstáculo que 
podemos definir como subjetivo en principio puede ser "el no atreverse» a iniciar el cambio de comportamiento. Veamos qué condiciona esa falta de «atrevimiento»:

Iniciar comportamientos transversales en su unidad supone que hay que arriesgarse a saltar barreras como el "principio de jerarquía», y no está claro que el discurso de la empresa a su favor sea homogéneo. Supone saber adónde les llevarán estos comportamientos, saber, por ejemplo, que no van a perder el puesto. Implica arriesgarse, sentirse capaces, tener imaginación, saber implicar a los trabajadores, superar los conflictos, romper la inercia, la comodidad, vencer el miedo al ridículo, pasar por alto las opiniones de los que piensan diferente... Supone que esté permitido equivocarse, fracasar, tener poder formal para tomar decisiones. Saber para qué cambiar los modos de hacer...

Tal cúmulo de condicionantes organizativos produce este criterio conductual, que hemos definido como "principio de negatividad organizativa»: "si me arriesgo, pierdo yo; si no me arriesgo, pierde la empresa». Principio que permite excluir todo problema que se refiera al "espacio común", que es precisamente el espacio del aprendizaje organizativo, la búsqueda de alternativas a las rutinas improductivas.

\section{Elucidar contradicciones}

Pero el obstáculo más grave es generar un cambio organizativo hacia la implicación cooperativa tras las experiencias de ajustes de plantilla con salidas indiscriminadas de la empresa de gente muy implicada en la gestión tradicional. Otro grave problema es la percepción de los efectos de las políticas de competitividad en la ausencia de salidas laborales de los hijos. Se introduce así el entorno, local y global, y el tiempo futuro como variables inevitables. De ahí que sean necesarios cambios en el modelo de gestión más allá de los aprendizajes estrictamente organizativos, y también que el auténtico aprendizaje organizacional quizás se dé solamente en el aprendizaje interorganizativo.

La atención para superar barreras la han focalizado, a la hora de empezar a andar, en los sistemas de reconocimiento y de retribución. Se considera una incoherencia que se mantengan sistemas de reconocimiento que premian la competitividad interna al mismo tiempo que se quieren generar conductas de cooperación. Se considera injusto que cobren igual los trabajadores participativos que los que no lo son, pero los convenios apenas permiten discriminar en este sentido. "Es preciso hallar un sistema motivador que sea capaz de motivar al que no participa», decía uno. Se percibe como necesario un sistema salarial variable, en una línea que discrimine lo que verdaderamente sean aportaciones objetivables en el tiempo, ya que existen experiencias de discriminaciones positivas a comportamientos aparentemente más productivos que en la práctica no lo son, con lo cual se corrompe el modelo y se generaliza el descontento. Son interminables los aspectos de este problema. No olvidemos los efectos ambiva- 
lentes de premiar conductas individuales y/o de grupos. Con esto ponemos en evidencia la problemática que encierra lo que sólo es un instrumento del cambio.

Siendo éste un grupo selecto, con alto interés por hacer bien las cosas en la empresa, que han sido capaces de cambiar su rol de mandos intermedios, nos interesa conocer lo que opinan sus trabajadores.

\section{Posibilitar el feed-back a todos los niveles}

Hemos iniciado una serie de encuentros y entrevistas con los trabajadores. No perciben que la polivalencia (consecuencia del aprendizaje organizativo) sea un aprendizaje ni que suponga ninguna mejora para ellos, ni personal, ni profesional, ni de status. Lo único que sí perciben como cambio es el trato que se les da ahora y las buenas relaciones con el que antes asumía las funciones de «encargado», hoy Jefe de Unidad. Entienden que el nuevo modelo interesa a la empresa como forma de sacarles mayor rendimiento. Su paradigma sigue siendo «empresa versus obreros» («curritos», se autodenominan, expresando así su significado en el conjunto) y «curritos de mono azul» versus «técnicos de batas blancas». Al que participa lo ven como «empresa». Se consideran, sin embargo, buenos obreros, profesional y personalmente hablando. De hecho, no están resistentes y relatan cómo resuelven desajustes tecnológicos debidos a la introducción de nuevas máquinas con auténticos aprendizajes llenos de iniciativa e ingenio, que, sin embargo, no son juzgados por ellos mismos como tales.

Con estos dos tipos de análisis, que son la proyección de dos estamentos que necesitan cooperar para aprender, se puede ver la complejidad y la envergadura del problema. Las organizaciones empresariales están sumidas en un mar de contradicciones. Sin embargo, cuando lees muchos de los libros de gestión, dan la impresión de que la realidad social fuera lineal y simple, ya preparada para el cambio, a través de estrategias e instrumentos meramente organizativos.

\section{La comunicación}

Asumir este tipo de información, junto con la información que procede del entorno, al más alto nivel organizativo necesariamente debe remover los esquemas mentales y los paradigmas que determinan el organizarse.

\section{El liderazgo}

Veamos una organización del segundo sector, el público. Podríamos referir varias experiencias de aprendizaje organizativo muy bien planteadas y bien 
ancladas en el poder, con excelentes lideres, que se han arriesgado a acometer cambios hacia la mejora de la calidad de los servicios y de la gestión. Son líderes con motivaciones sociales y profesionales básicamente (es decir, no retributivas). La cultura burocrática es un problema a resolver, de eso se trata. El problema del problema es que la percepción de las ventajas y de los valores está determinada, en su mayor parte, por la cultura mercantilista. No se perciben las ventajas y valores de lo público. Sólo se perciben niveles y diferencias salariales. A pesar de todo, se llegan a generar sinergias en torno a valores coherentes con la institución. El problema aquí es la falta de cultura política, que dificulta una adecuada relación entre el poder técnico y el poder político, que permita una continuidad en estos aprendizajes.

Las organizaciones del nuevo tipo necesitan lideres de nuevo estilo, que serán los líderes del siglo XXI. Hay muy buenos aprendizajes de este tipo, que son parte del aprendizaje organizativo — organizacional- Es preciso superar la política con minúsculas y recuperar la POLITICA con mayúsculas. El poder no es unilateral ni unidimensional, sino recíproco y multidimensional. El aprendizaje organizacional suma poder, no resta.

\section{La interdisciplinariedad}

No obstante, el propio nivel técnico-organizativo del sector público tiene también problemas semejantes a los analizados en la empresa. Podríamos referirnos a un servicio que necesita trabajar en equipo para integrar funciones y saberes disciplinares complementarios, como garantía de calidad para el usuario. Es necesario, pues, generar el «espacio de cooperación», que debe integrar diferentes categorías, diferentes funciones y diferentes saberes técnicos, además de diferentes personalidades. La expectativa de aprendizajes es clara: mejorará la calidad del servicio, se podrán elaborar y gestionar aprendizajes como productos del grupo (metodología sistematizada, casos paradigmáticos, conocimientos interdisciplinares acerca del objeto, modelos de intervención, etc.) y también enriquecimiento personal y profesional. En la práctica, las diferencias se viven como ventajas o desventajas individuales, no como complementarias. Las soluciones informales y ocasionales que ocurren son aquí denominadas "parcheos".

\section{EL SABER ACADEMICO Y LA FORMACION DE PROFESIONALES}

Un problema muy grave que el propio grupo no confiesa es que las distintas «voces» disciplinares están vacías de contenido de cara al trabajo interdisciplinar. "La Universidad no enseña a trabajar en equipo", dicen algunos para expresar eso mismo. El verdadero significado de ese hecho, además de la ausen- 
cia de hábitos y habilidades de debate y de construcción de sinergias de grupo, es que el saber disciplinar transmitido es reduccionista, parcelado, y ciego respecto a su potencia cognitiva, ya que no ha elucidado y fundamentado sus propios paradigmas y, sobre todo, desconoce otros paradigmas que abordan su mismo objeto desde otra dimensión. El saber académico adolece de los mismos problemas que el saber y el hacer organizativos. Quizá siga siendo necesario el parcelamiento disciplinar, pero sin perder jamás su nivel teórico más noble, y su capacidad integradora de saberes alternativos. Hay que superar, a través de la inteligencia organizativa, el efecto del especialismo, que conlleva pérdida de capacidad cognitiva. El último «bucle» del aprendizaje, irrenunciable desde la inteligencia de los procesos, es el epistémico, también indispensable en el saber organizativo.

Es una llamada de atención para el modelo de transmisión y generación de conocimientos de la Universidad. Parece que los tiempos están poniendo en evidencia la necesidad de volver a la síntesis cognitiva de donde partió el saber. Ahora, a partir de la profundización que permite la especialización. Seguramente el verdadero lugar de la Universidad es el tercer ciclo, no sólo el doctorado, sino la formación de postgrado interdisciplinar y vinculada al campo de aplicación, contextualizado. Objetivo que exige planteamientos superiores al pragmatismo inmediato con el que se orientan los saberes profesionales.

\section{LA GENERACION DE COMPETENCIAS ORGANIZATIVAS}

El aprendizaje organizativo es fundamental para todo tipo de organización. La tarea es ardua y urgente. Y no se resuelve sólo con instrumentos y técnicas. Como bien dice Senge, el aprendizaje a alcanzar es una metanoia. Un cambio significativo en la relación de los actores, además de un cambio en su modo de pensar. Senge pide pensamiento sistémico, nosotros añadimos: y dialéctico. Crozier identifica ese cambio como capacidad de generar y cambiar las reglas del juego entre los actores. Habermas, desde otra óptica, como desarrollo de la capacidad comunicativa, que, a su vez, permitirá la redefinición de la racionalidad y la legitimidad operantes. Touraine dice que una toma de conciencia organizativa supone aumentar el nivel de intervención de una sociedad sobre sí misma, para lo cual hay que reintegrar racionalización (en el sentido técnicoinstrumental) con otras dimensiones de la acción, de la organización social y de los mismos actores.

En la generación y gestión de competencias hay que contemplar, efectivamente, todas las dimensiones de la acción social: la realidad objetiva, que requiere competencias técnico-científicas; la social, que requiere comprensión de los procesos organizativos como espacios generadores de normas que determinan los comportamientos, y la subjetiva, que se configura como personalidad y que determina la percepción de la realidad y que es el soporte de las 
facultades y capacidades necesarias para que las tres dimensiones se integren racionalmente, es decir, objetiva y comprehensivamente.

Quiere esto decir que las competencias organizativas son algo más que técnicas. Exigen conocimientos que permitan la comprensión global para la actuación local en situaciones de gran complejidad e incertidumbre.

No basta con generar y acumular conocimientos, proceso ya difícil, como hemos visto, en tanto que son transversales y provienen del trabajo interfuncional; hay que transferirlos a la organización y transformarlos en tecnologías y habilidades capaces de resolver los nuevos problemas organizativos.

En otro lugar hicimos referencia a una espiral de aprendizaje organizativo que, partiendo de la resolución de problemas de tipo técnico, asume problemas que, no resueltos a ese nivel, requieren toma de decisiones por parte de actores diversos, que, a su vez, deben asumir el conflicto que supone no ser capaces de resolver el problema, aparentemente técnico, debiendo aclarar posiciones y negociar, para, finalmente, ascender a un plano "metaparadigmático», donde poder confrontar los diversos paradigmas y esquemas mentales que determinan sus rígidos "posicionamientos», para dilucidar, objetivamente, la funcionalidad de dichos paradigmas y esquemas. Es una espiral por cuanto es un proceso de ascenso para descender al nivel operativo, listos para ascender de nuevo cuando la situación lo exija.

Ese tipo de aprendizaje, que no supone sólo un «saber cómo», sino que constituye un "saber qué», pero que sobre todo supone un aprender a aprender, es la base para mantener abiertos los diferentes subsistemas de la organización y para flexibilizarlos, incluida la propia estrategia externa e interna. No olvidemos que el problema de base es la innovación.

De los ejemplos que pusimos más arriba podemos deducir que el tipo de aprendizaje de que hablamos aparece como "contracultural» en la organización, y que, superadas las barreras estructurales, lo que debe producirse es un desaprendizaje. Aprender a desaprender es la competencia cognitiva más compleja y difícil, que requiere otro nivel o perspectiva sobre la realidad. Es uno de los indicadores de inteligencia más definitivos.

Llegados a ese nivel podemos esperar las conductas cooperativas creativas. Siempre que la estructura organizativa lo permita. El trabajo creativo necesita un contexto organizativo que le provea de medios, que le permita cometer errores —excelente campo de aprendizaje_-, que le permita experimentar, trabajar en hipótesis, abrirse a otras fuentes de información, contrastar su saber, etcétera. Es preciso un tipo de poder y de autoridad coherentes con el trabajo altamente profesionalizado. Abrir el poder para que los profesionales puedan compartirlo y ver así las ventajas del valor que añaden a la organización. Profesionalizar también la autoridad para que sea capaz de generar un sistema autonómico, que dé confianza a los actores, para que puedan surgir los antagonismos y el abordaje conjunto de los problemas nuevos. 


\section{ESTRATEGIAS DE APRENDIZAJE ORGANIZATIVO}

En un plano más pragmático podríamos sugerir estrategias de intervención a favor del aprendizaje organizativo y organizacional que propicien el contraste, el debate, las visiones alternativas, la dinámica frente a la inercia, como es la metodología paradójica, o el trabajo con las analogías metafóricas, que colocan al actor en una perspectiva diferente a la habitual, provocando el trabajo en hipótesis y la experimentación. Enfocar la gestión hacia el aprendizaje es orientar la cultura organizativa hacia la investigación.

Estas son algunas estrategias paradójicas que se han hecho familiares en los nuevos modelos de gestión: para tratar lo local, transferir el problema a lo global. Ver las crisis o amenazas como oportunidades. Enfrentar la lentitud del sistema con la aceleración del cambio en el entorno o en una variable significativa. Ante la incertidumbre, mayor autonomía e iniciativa. Frente a rigidez del saber organizativo, mayor información. Contra la confusión, mostrar un camino a construir (proyecto). Frente al simplismo, complejidad. Contra la pasividad, «masas críticas». Cuanto menos estructura, más sinergia. Cuanto menos poder formalizado, más poder organizativo. Contra la estanqueidad de la muerte, el desorden de la vida. Contra aburrimiento y rutina, tensión creadora, problemas...

Desde el punto de vista estructural, es la organización por proyectos o productos la que más facilita la integración de funciones y su orientación al proceso global, lo cual permite a cada actor entender y evaluar su aportación al conjunto.

Se trata, en definitiva, de hacer emerger la motivación intrinseca, propia de la conducta de aprendizaje creativo, altamente satisfactoria, que se autoalimenta y es de duración indefinida. Si se consigue esa motivación, junto con la implicación en la organización, se han sentado las bases para la organización que aprende.

\section{BIBLIOGRAFIA}

ARGYRIS, C., y SCHÖN, D. (1978): Organizational Learning: a theory of action perspective, Reading (Massachusetts), Addison-Wesley.

Aubrey, R., y COHEN, P. (1995a): Working wisdon: timeless skills and vanguard strategies for learning organizations, San Francisco, Jossey-Bass Publisher.

- (1995b): La organización en aprendizaje permanente. Estrategias prácticas para ganar ventajas competitivas. Bilbao, Ed. Deusto.

Cohen, M. D., y Sproull, L. S. (eds.) (1995): Organizational Learning, Londres, SAGE Publications.

Crozier, M. (1989a): L'entreprise a l'ecoute. Apprendre le management post-industriel, París, Inter Editions.

- (1989b): "Obstáculos burocráticos a la innovación», en III Foro de Issyk-Kull, UNESCO, Resistencia a la innovación en sistemas complejos, Madrid, Instituto de Ciencias del Hombre.

GEISSLER, H. (1991): Vom Lernen in der Organisation, vom Lernen der Organisation, Hamburgo. 
Habermas, J. (1981): Theorie des komunikativen Handelns, Frankfurt, Suhrkamp Verlag.

- (1987): Teoría de la acción comunicativa, Madrid, Taurus.

Landier, H. (1991): Vers l'entreprise intelligente, París, Calmann-Lévy. Traducción (1992): Hacia la empresa inteligente. Guía para la gestión del cambio, Bilbao. Ed. Deusto.

Lapassade, G. (1974): Groupes, organisations, intitutions, París, Bordas. Traducción (1977): Grupos, organizaciones e instituciones. La transformación de la burocracia, Barcelona, Granica Ed.

Moingeon, B., y Edmondson, A. (1996): Organizational Learning and Competitive Advantage, Londres, SAGE Publications.

Moreno PelÁez, F. (1981): Hombre y sociedad en el pensamiento de Fromm, México, FCE (contiene bibliografía básica sobre aprendizaje social).

- (1995): «Presupuestos teóricos y perspectivas del DO», Actas del V Congreso Español de Sociología, Granada.

- (1996): "Gestión de la toma de conciencia organizativa", en la Memoria del III Congreso Internacional sobre Desarrollo Organizacional y Calidad Total, Univ. de Lima, Perú.

Morgan, G. (1993): Imaginization. The art of creative management, Londres, SAGE Publications.

Morin, E. (1986): Le Methode. III: La connaissance de la conaissance, París, Editions du Seuil. Traducido por Cátedra, Madrid, 1988.

Peters, T. (1994): The Tom Peters seminar: crazyt times call for crazy oganizations, N.Y., Vintage Books. Traducción (1994): Nuevas organizaciones en tiempos de caos, Bilbao, Ed. Deusto.

Revilla Gutiérrez, E. (1995): Factores determinantes del Aprendizaje Organizativo. Un modelo de Desarrollo de Productos, Valladolid, Club Gestión de la Calidad y Universidad de Valladolid (Ppremios 1996: a la mejor Tesis Doctoral).

Senge, P. M. (1990): The Fifth Discipline, N.Y., Doubleday. Traducción (1992): La Quinta Disciplina. El arte y la práctica de la organizción abierta al aprendizaje, Barcelona, Granica.

SÉRIEYX, H. (1993): Le big bang des organisations, París, Calmann-Lévy. Traducción (1994): El big bang de las organizaciones, Barcelona, Ediciones $\mathrm{B}$.

STAhl, T.; NyHAN, B., y D’AlOjA, P. (1993): La organización cualificante (The Learning Organization), Comisión de las Comunidades Europeas: Eurotecnet.

Touraine, A. (1987): «Modernidad y especificidad culturales», en Resistencia a la innovación en sistemas complejos (antes referido).

\section{NUMEROS MONOGRAFICOS DE REVISTAS SOBRE EL TEMA}

Revista de ADITE F.I.T. Número especial, junio 1995, dedicado a De l'organisation formatrice a l'organisationa apprenante.

Revista del CEDEFOP, FORMACION PROFESIONAL. Número 5, mayo-agosto 1995/II, dedicado a La gerenaración de competencias en la empresa.

Revista EDUCATION PERMANENTE. Número 112, de octubre 1992, dedicado a L'organisation qualifiante. 


\begin{abstract}
Organisational Learning amounts to more than generating skills in the members of a particular organisation. It consists of organisational breakthroughs (in terms of methods, technologies, culture, products) and has the effect of improving results and strengthening the dialectical relationship with the environment in such a way as to increase the organisation's survival and development prospects. These improvements are obtained through what we might call organisational-institutional learning, a form of training geared to generating a "cooperative area» that surpasses organisational barriers and internal competitiveness by creating niches of knowledge and turning them into specific power bases. Two methodological approaches are therefore required to tackle this problem: systematic and dialectic reflection.
\end{abstract}

